\title{
2-Deoxy-D-glucose initiates hepatocyte differentiation in human induced pluripotent stem cells
}

\author{
MINORU TOMIZAWA ${ }^{1}$, FUMINOBU SHINOZAKI ${ }^{2}$, YASUFUMI MOTOYOSHI ${ }^{3}$, \\ TAKAO SUGIYAMA $^{4}$, SHIGENORI YAMAMOTO ${ }^{5}$ and NAOKI ISHIGE ${ }^{6}$ \\ Departments of ${ }^{1}$ Gastroenterology, ${ }^{2}$ Radiology, ${ }^{3}$ Neurology, ${ }^{4}$ Rheumatology, ${ }^{5}$ Pediatrics and ${ }^{6}$ Neurosurgery, \\ National Hospital Organization, Shimoshizu Hospital, Yotsukaido, Chiba 284-0003, Japan
}

Received March 27, 2016; Accepted February 15, 2017

DOI: $10.3892 / \mathrm{mmr} .2017 .6405$

\begin{abstract}
To initiate hepatocyte differentiation in human induced pluripotent stem (iPS) cells, cells are cultured in a medium lacking glucose but supplemented with galactose (hepatocyte selection medium, HSM) or in medium supplemented with oncostatin $\mathrm{M}$ and small molecules (hepatocyte differentiation inducer, HDI). In the present study, 2-Deoxy-D-glucose (2DG), an analogue of glucose, was utilized instead of glucose deprivation and the effect of 2DG supplementation on iPS differentiation was examined. First, 201B7 cells, an iPS cell line, were cultured in HSM or HDI media for 2 days and then subjected to reverse transcription-quantitative polymerase chain reaction (RT-qPCR) in order to analyze expression levels of established hepatocyte markers, including cytosolic aspartate aminotransferase (AST), mitochondrial AST, alanine aminotransferase (ALT), and glycerol kinase. mRNA expression levels of mitochondrial AST, ALT, and glycogen synthase significantly increased following culture in HSM and HDI compared with ReproFF media. Cytosolic AST mRNA expression levels significantly increased following culture in HDI compared with ReproFF media, but not in HSM. To test the effect of 2DG on iPS differentiation, 201B7 cells were cultured in ReproFF, a feeder-free medium that retains pluripotency, supplemented with 2DG. Following 7 days of culture, the cells were subjected to RT-qPCR to analyze expression levels of $\alpha$-fetoprotein (AFP), a marker of immature hepatocytes. AFP mRNA expression levels significantly increased with the addition of $0.1 \mu \mathrm{M} 2 \mathrm{DG}$ in the media, and galactose addition acted synergistically with 2DG to further upregulate AFP expression. In conclusion, the present study demonstrated that hepatocyte differentiation was initiated in iPS cells cultured in HSM and HDI media and
\end{abstract}

Correspondence to: Dr Minoru Tomizawa, Department of Gastroenterology, National Hospital Organization, Shimoshizu Hospital, 934-5 Shikawatashi, Yotsukaido, Chiba 284-0003, Japan E-mail: nihminor-cib@umin.ac.jp

Key words: 2-Deoxy-D-glucose, $\alpha$-fetoprotein, aspartate aminotransferase, alanine aminotransferase, glycerol kinase that 2DG could be used as a supplement instead of glucose deprivation to initiate hepatocyte differentiation in iPS cells.

\section{Introduction}

Hepatic failure, a condition where the number of functioning hepatocytes significantly decreases, is fatal (1). Transplantation of hepatocytes in patients with liver failure could be a promising treatment. Human induced pluripotent stem (iPS) cells are established with the introduction of reprogramming factors $(2,3)$. iPS cells are an ideal source for the generation of hepatocytes. The current protocols for generating hepatocytes from iPS cells consist of sequential stimulation with growth factors and introduction of transcription factors (4-9). The generated hepatocytes remain in an immature state, namely hepatic progenitor-like cells (10).

Aspartate aminotransferase (AST) is expressed in the cytosol (cytosolic AST) and mitochondria (mitochondrial AST) in myocytes and hepatocytes (11). AST catalyzes the interconversion of aspartate and $\alpha$-ketoglutarate to glutamate and oxaloacetate. Alanine aminotransferase (ALT) is mainly expressed in hepatocytes and catalyzes the conversion of pyruvate and glutamate to L-alanine and a-ketoglutarate (12). Glycogen synthase synthesizes glycogen from glucose metabolites (13). If a cell expresses AST, ALT, and glycogen synthase, it is considered to have a metabolic function specific to that of hepatocytes.

Glucose is indispensable for the survival of cells. Hepatocytes express galactokinase, an enzyme that catalyzes the conversion of galactose to galactose-1-phosphate. Galactose-1-phosphate enters the glycolytic pathway and is used as a source of energy by the cells. Hepatocyte selection medium (HSM), a medium that lacks glucose but contains galactose, is used to eliminate iPS cells and specifically select for hepatocytes (14). Hepatocytes survive in HSM because they express galactokinase, allowing them to utilize the galactose in the medium for their energy needs. Expression of $\alpha$-fetoprotein (AFP), a marker of immature hepatocytes, is increased in iPS cells cultured in HSM (15), suggesting that glucose deprivation and galactose supplementation promote hepatocyte differentiation of iPS cells.

2-Deoxy-D-glucose (2DG) is an analogue of glucose (16), which is taken up by cells similar to glucose. However, unlike 
glucose, it is not metabolized and it is accumulated in the cells. 2DG is used in positron emission tomography for diagnostic imaging of cancer (17). It is hypothesized that if 2DG was added in a medium, the cultured cells would take up 2DG similar to glucose, but the cells would not be able to metabolize 2DG as a source of energy. Such addition of 2DG in the medium would therefore be similar to glucose deprivation. In the present study, the effect of galactose and 2DG was examined on hepatocyte differentiation of iPS cells, by evaluating the expression of AFP, as a marker of immature hepatocytes. In addition, the expression levels of AST, ALT, and glycogen synthase in iPS cells cultured in HSM or hepatocyte differentiation inducer (HDI) were examined.

\section{Materials and methods}

Cell culture. A human iPS cell line, 201B7 (Riken BioResource Center, Tsukuba, Japan), was cultured on $10 \mathrm{~cm}$ dishes coated with Matrigel (BD Biosciences, Franklin Lakes, NJ, USA) in ReproFF medium (ReproCELL, Inc., Yokohama, Japan) at $37^{\circ} \mathrm{C}$ with $5 \%$ carbon dioxide in a humidified chamber. Once confluent, the cells were rinsed with PBS and harvested using Accutase (Innovative Cell Technologies, Inc., San Diego, CA, USA). The cells were then observed under a microscope (CKX41N-31 PHP; Olympus Corporation, Tokyo, Japan).

Reagents. Non-essential amino acids solution (NEAA; 100x) and sodium pyruvate $(100 \mathrm{x})$ were purchased from Thermo Fisher Scientific, Inc. (Waltham, MA, USA). The apoptosis inhibitor M50054 [2,2'-methylenebis (1,3-cyclohexanedione)] was purchased from Merck KGaA (Darmstadt, Germany). FPH1 [2-(N-(5-chloro-2-methylphenyl) methylsulfonamido)-N-(2,6-difluorophenyl) acetamide] was purchased from Xcessbio Biosciences, Inc. (San Diego, CA, USA) (18). Galactose, ornithine, glycerol, oncostatin M, nicotinamide, proline and L-glutamine were purchased from Wako Pure Chemical Industries, Ltd. (Osaka, Japan). 2DG was purchased from Sigma-Aldrich; Merck KGaA.

HSM and HDI media. 201B7 cells were cultured in 6-well plates coated with Matrigel (BD Biosciences) in HSM or HDI for 2 days and then subjected to reverse transcription-quantitative polymerase chain reaction (RT-qPCR). HSM consisted of Leibovitz's-15 (L15) medium (Thermo Fisher Scientific, Inc.), with the omission of arginine, tyrosine, glucose, and sodium pyruvate and the addition of galactose $(900 \mathrm{mg} / \mathrm{l})$, ornithine $(1 \mathrm{mM})$, glycerol $(5 \mathrm{mM})$, and proline $(260 \mathrm{mM})(14)$. Proline (30 mg/l) was added because it is required for DNA synthesis (19). Aspartic acid was not included because it is one of the products of ornithine metabolism and a substrate for arginine synthesis. KnockOut serum replacement (Thermo Fisher Scientific, Inc.) was added at a final concentration of $10 \%$ and was used instead of fetal calf serum to establish xeno-free, defined conditions. HDI medium was prepared using oncostatin M (20 ng/ml), FPH1 (20 $\mu \mathrm{M})$, M50054 (100 mg/l), NEAA (1x), sodium pyruvate (1x), nicotinamide $(1.2 \mathrm{mg} / \mathrm{ml})$, proline $(30 \mathrm{ng} / \mathrm{ml})$, and L-glutamine $(0.3 \mathrm{mg} / \mathrm{ml})$. Proline and nicotinamide are required for proliferation of primary hepatocytes $(19,20)$.
Conventional media. $201 \mathrm{~B} 7$ cells were harvested with Accutase, and transferred to a $15 \mathrm{ml}$ tube (Asahi Glass, Tokyo, Japan). The cells were centrifuged at $100 \mathrm{x} g$ for $3 \mathrm{~min}$ at $4^{\circ} \mathrm{C}$. The cells were spread onto 6-well plates (Asahi Glass) coated with Matrigel at the density of $10^{6}$ cells for each well and were cultured in ReproFF, L15, William's E (WE) (Thermo Fisher Scientific, Inc.), or Dulbecco's modified Eagle's medium/Ham's F-12 nutrient mixture (DF12; Sigma-Aldrich; Merck KGaA). L15, WE, and DF12 were supplemented with $1.2 \mathrm{mg} / \mathrm{ml}$ nicotinamide, $30 \mathrm{ng} / \mathrm{ml}$ proline, and $10 \%$ Knockout serum replacement. Nicotinamide and proline were added because they are required for proliferation of primary hepatocytes $(19,20)$. For some experiments, $2 \mathrm{DG}$ at a concentration of $0,0.1,1$ or $10 \mu \mathrm{M}$ was added and galactose at a concentration of $900 \mathrm{mg} / \mathrm{ml}$ was added, as indicated. The cells were cultured for 7 days and then subjected to RT-qPCR.

$R T-q P C R$. Total RNA (5 $\mu \mathrm{g})$, isolated with Isogen (Nippon Gene Co., Ltd., Tokyo, Japan), was used for first-strand cDNA synthesis with SuperScript III reverse transcriptase and oligo (dT) primers (Thermo Fisher Scientific, Inc.), following the manufacturer's instructions. Total RNA from human fetal liver and adult liver was purchased from Clontech Laboratories, Inc. (Mountainview, CA, USA). qPCR was performed in a volume of $20 \mu \mathrm{l}$ for 40 cycles of two steps consisting of a 5 sec denaturation step and a $5 \mathrm{sec}$ annealing-extension step, using Fast SYBR Green Master Mix (Thermo Fisher Scientific, Inc.) and the results were analyzed using the MiniOpticon Real-Time PCR system (Bio-Rad Laboratories, Inc., Hercules, CA, USA). The primer sequences are listed in Table I. RPL19 was used as an endogenous reference control because it is a constitutively expressed housekeeping gene (21). Gene expression levels were analyzed automatically using the MiniOpticon system based on the $2^{-\Delta \Delta \mathrm{Cq}}$ method (22). The relative expression was calculated as the expression level of a specific gene divided by that of RPL19.

Statistical analysis. Relative expression levels of genes were analyzed by one-factor analysis of variance using JMP version 5.0J software (SAS Institute, Inc., Cary, NC, USA). Student's t-test was used as a post hoc test. $\mathrm{P}<0.05$ was considered to indicate a statistically significant difference.

\section{Results}

To analyze the effect of culturing iPS cells in different media on the expression levels of liver-specific genes, 201B7 cells were cultured in ReproFF, HSM, or HDI for 2 days. RNA was isolated and subjected to RT-qPCR in order to determine the mRNA expression levels of cytosolic AST (Fig. 1A), mitochondrial AST (Fig. 1B), ALT (Fig. 1C) and glycogen synthase (Fig. 1D). The mRNA expression levels of mitochondrial AST, ALT, and glycogen synthase significantly increased by culture in HSM and HDI compared with ReproFF media (Fig. 1B-D). The expression level of cytosolic AST significantly increased by culture in HDI compared with ReproFF media, but not in HSM media (Fig. 1A). These results suggest that the absence of glucose or arginine and the addition of galactose and ornithine increased the expression levels of liver-specific genes and glycogen synthase. Expression levels of each gene were 
Table I. Sequences of primers used in the present study.

\begin{tabular}{|c|c|c|c|}
\hline Gene & Sequence (5'-3') & Product size (bp) & GenBank accession no. \\
\hline AFP & $\begin{array}{l}\text { F-ACACAAAAAGCCCACTCCAG } \\
\text { R-GGTGCATACAGGAAGGGATG }\end{array}$ & 147 & NM_001134 \\
\hline Cytosolic AST & $\begin{array}{l}\text { F-GATGGAGAAGATCGTGCGGAT } \\
\text { R-AATCCGGTCAGCCATTGTCTT }\end{array}$ & 133 & M37400 \\
\hline Mitochondrial AST & $\begin{array}{l}\text { F-ACATGTAGTGACACAGGGCAG } \\
\text { R-CACGAGGAACCTGACACTTCA }\end{array}$ & 192 & M22632 \\
\hline ALT & $\begin{array}{l}\text { F-GAGCCCACTGTACTTGCTCTT } \\
\text { R-TTTTCCTGGAGTCAGACTGCC }\end{array}$ & 113 & ВC018207 \\
\hline GS & $\begin{array}{l}\text { F-ATATCCCAGGCCTTCCTCAGT } \\
\text { R-CGTGGCTCAGTGAAAATGGTG }\end{array}$ & 164 & S70004 \\
\hline RPL19 & $\begin{array}{l}\text { F-CGAATGCCAGAGAAGGTCAC } \\
\text { R-CCATGAGAATCCGCTTGTTT }\end{array}$ & 157 & ВC095445 \\
\hline
\end{tabular}

Primers were designed to have an annealing temperature of $60^{\circ} \mathrm{C}$ and quantitative polymerase chain reaction was performed for 40 cycles. F, forward; R, reverse; AFP, $\alpha$-fetoprotein; AST, aspartate aminotransferase; ALT, alanine aminotransferase; GS, glycogen synthase; RPL19, ribosomal protein $\mathrm{L} 19$.

A

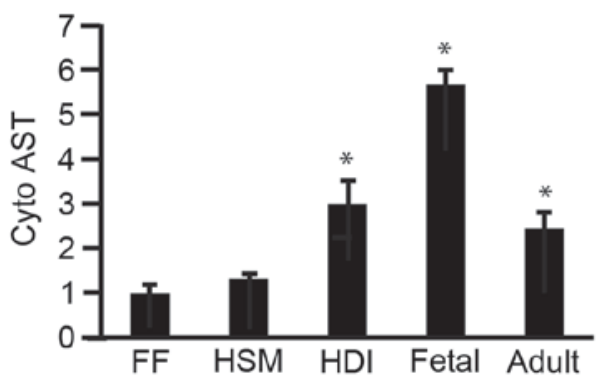

C

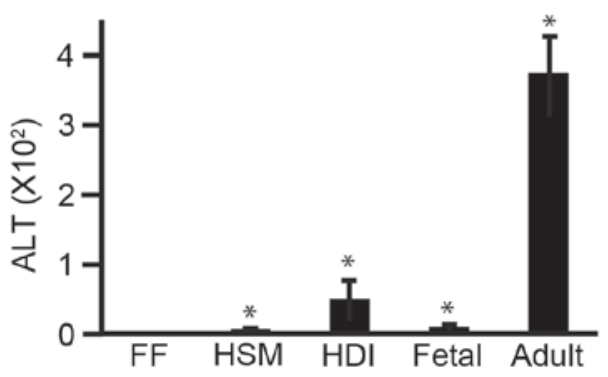

B

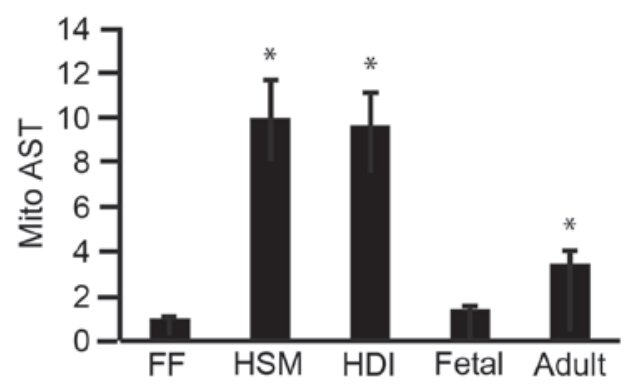

D

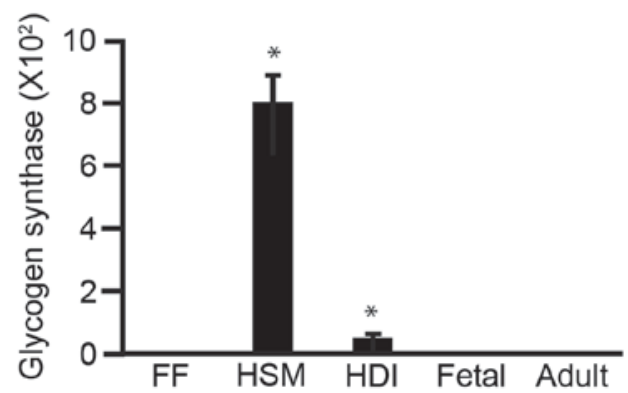

Figure 1. Effect of different culture media on the expression of liver-specific genes. $201 \mathrm{~B} 7$ cells were cultured in FF, HSM, or HDI media for 2 days. The cells were then subjected to reverse transcription-quantitative polymerase chain reaction to analyze the mRNA expression levels of (A) Cyto AST, (B) Mito AST, (C) ALT and (D) glycogen synthase. "P<0.05 compared with FF (n=3). FF, ReproFF; HSM, hepatocyte selection medium; HDI, hepatocyte differentiation inducer; Cyto, cytosolic; AST, aspartate aminotransferase; Mito, mitochondrial; ALT, alanine aminotransferase; Fetal, fetal liver total RNA; Adult, adult liver total RNA.

analyzed with fetal and adult liver to compare with those of HSM and HDI and identify whether iPS cells acquired expression of liver-specific genes. Cytosolic and mitochondrial AST were expressed in HSM and HDI at comparable levels to fetal and adult liver.

To address the possibility that galactose affected hepatocyte differentiation of 201B7 cells, the cells were cultured in L15, WE, and DF12 media in the absence or presence of galactose and then total RNA was isolated and subjected to RT-qPCR (Fig. 2). The mRNA expression levels of AFP, a marker of immature hepatocytes, were significantly higher in the L15, WE and DF12 media compared with the ReproFF media $(\mathrm{P}<0.05$; Fig. 2). However, for each medium, mRNA expression levels of AFP were similar in the presence or absence of galactose (Fig. 2). These results suggest that galactose had no effect on the differentiation of 201B7 cells to the hepatocyte lineage. 


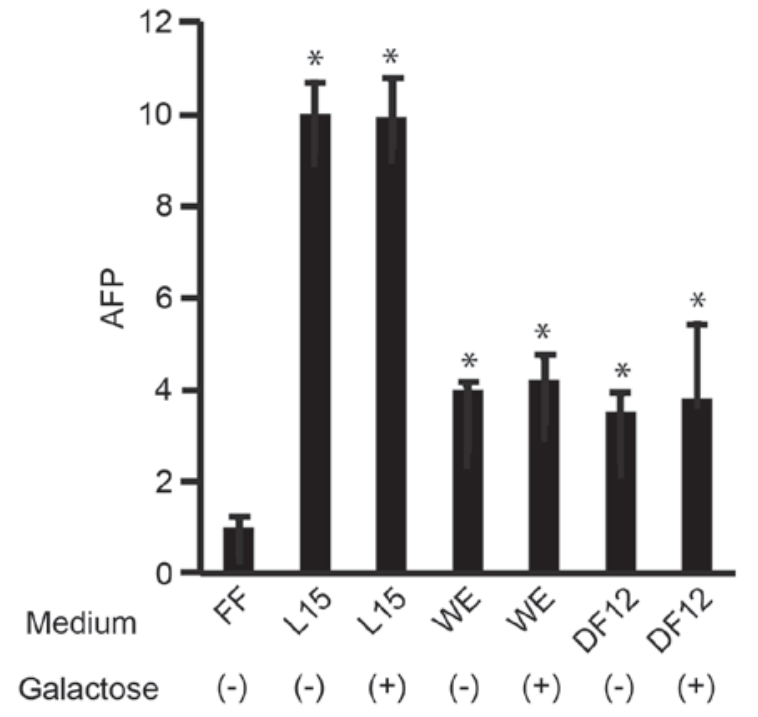

Figure 2. Effect of galactose on expression of the AFP hepatocyte marker. $201 \mathrm{~B} 7$ cells were cultured in FF, L15, WE, or DF12 media with (+) or without (-) galactose $(900 \mathrm{mg} / \mathrm{ml})$. The cells were then subjected to reverse transcription-quantitative polymerase chain reaction to analyze the mRNA expression levels of AFP. "P<0.05 compared with FF ( $n=3)$. AFP, $\alpha$-fetoprotein; FF, ReproFF; L15, Leibovitz's-15; WE, William's E; DF12, Dulbecco's modified Eagle's medium/Ham's F-12 nutrient mixture.



Figure 3. Effects of 2DG and galactose on expression of the AFP hepatocyte marker. 201B7 cells were cultured in FF medium with (+) or without (-) galactose $(900 \mathrm{mg} / \mathrm{ml})$ and/or 2DG $(0.1-10 \mu \mathrm{M})$. The cells were then subjected to reverse transcription-quantitative polymerase chain reaction to analyze the expression level of AFP. "P $<0.05$ compared with FF and ${ }^{\text {" }} \mathrm{P}<0.05$ compared with $0.1 \mu \mathrm{M}$ 2DG (n=3). 2DG, 2-Deoxy-D-glucose; AFP, $\alpha$-fetoprotein; Fetal, fetal liver total RNA.

To analyze the effect of the glucose analogue 2DG on hepatocyte differentiation of 201B7 cells, the cells were cultured in ReproFF medium in the absence or presence of 2DG and/or galactose. Total RNA was then isolated and subjected to RT-qPCR to determine the mRNA expression levels of AFP (Fig. 3). The mRNA expression levels of AFP significantly increased with the addition of $0.1 \mu \mathrm{M} 2 \mathrm{DG}$ (Fig. 3). In the absence of galactose, AFP was upregulated with 0.1 and $10 \mu \mathrm{M}$ but not with $1.0 \mu \mathrm{M}$ of $2 \mathrm{DG}$. It was not clear why expression level of AFP with $1.0 \mu \mathrm{M}$ of $2 \mathrm{DG}$ did not increase. The expression levels of AFP increased at $0.1 \mathrm{mM}$ of 2DG with galactose more than without it. Again, the reason was not clear, but it may be speculated that mechanism of galactose metabolism is involved with hepatocyte differentiation. Addition of galactose acted synergistically with the $0.1 \mu \mathrm{M} 2 \mathrm{DG}$ treatment to further increase mRNA expression of AFP in 201B7 cells (Fig. 3). These results suggested that 2DG and galactose promoted hepatocyte differentiation of 201B7 cells.

\section{Discussion}

Glucose is an indispensable component for cell survival. During somatic cell reprogramming, energy production shifts from an oxidative state to a glycolytic state (23). The source of energy depends on glycolysis in iPS cells (24). Galactose is metabolized through glycolysis following conversion to galactose-1-phosphate by galactokinase. iPS cells do not survive in either HSM or HDI media. In the present study, culturing the iPS cell line 201B7 in either HSM or HDI media resulted in an increase of the expression levels of AFP, suggesting the initiation of iPS cell differentiation to the hepatocyte lineage $(15,25)$. AST, ALT, and glycogen synthase are markers of hepatic function (26). In the present study, the expression levels of AST, ALT, and glycogen synthase increased by culture of 201B7 cells in HSM and HDI media, confirming that hepatocyte differentiation was initiated in iPS cells by these media. Tomizawa et al and Phillips et al $(25,26)$ have established a medium based on L15 that lacks glucose and contains galactose, that promotes the differentiation of iPS cells to the hepatocyte lineage. These previous reports, together with the present results, suggest that hepatocyte differentiation is initiated in iPS cells cultured in media lacking glucose and supplemented with galactose. The detailed mechanism of this, however, remains unclear.

Next, the aim was to identify whether glucose deprivation or galactose supplementation was responsible for the initiation of hepatocyte differentiation of iPS cells. The present results demonstrated that galactose supplementation did not affect the expression level of AFP. This suggests that galactose supplementation was not involved in hepatocyte differentiation of iPS cells.

A major problem with HSM and HDI media is that iPS cells do not survive for $>3$ and $>7$ days, respectively. To overcome this limitation, 2DG was used instead of glucose deprivation. The results demonstrated that 2DG supplementation in the media resulted in a significant increase in the mRNA expression levels of AFP in 201B7 cells. Moreover, galactose supplementation enhanced the 2DG-initiated AFP upregulation. These data suggest that the addition of 2DG and galactose in the media initiated hepatocyte differentiation of iPS cells.

A limitation of the present study was that analysis of hepatocyte differentiation was mainly based on determination of mRNA expression of only one hepatocyte marker, namely AFP. Further studies with immunostaining for hepatocyte-specific markers, such as AFP and albumin, and with functional analysis such as indocyanine green uptake, will be required in the future to further confirm the present results.

In conclusion, the present study demonstrated that hepatocyte differentiation was initiated in iPS cells by culturing in HSM and HDI media. The present results suggest that 2DG may be used as a supplement instead of glucose deprivation to initiate hepatocyte differentiation in iPS cells. 


\section{Acknowledgements}

The present study was supported by a Grant-in-Aid for Scientific Research from the Japan Society for the Promotion of Science (grant no. 15K09032).

\section{References}

1. Sugawara K, Nakayama N and Mochida S: Acute liver failure in Japan: Definition, classification, and prediction of the outcome J Gastroenterol 47: 849-861, 2012.

2. Takahashi K, Tanabe K, Ohnuki M, Narita M, Ichisaka T, Tomoda K and Yamanaka S: Induction of pluripotent stem cells from adult human fibroblasts by defined factors. Cell 131: 861-872, 2007

3. Hirschi KK, Li S and Roy K: Induced pluripotent stem cells for regenerative medicine. Annu Rev Biomed Eng 16: 277-294, 2014.

4. DeLaForest A, Nagaoka M, Si-Tayeb K, Noto FK, Konopka G, Battle MA and Duncan SA: HNF4A is essential for specification of hepatic progenitors from human pluripotent stem cells. Development 138: 4143-4153, 2011.

5. Si-Tayeb K, Noto FK, Nagaoka M, Li J, Battle MA, Duris C, North PE, Dalton S and Duncan SA: Highly efficient generation of human hepatocyte-like cells from induced pluripotent stem cells. Hepatology 51: 297-305, 2010

6. Song Z, Cai J, Liu Y, Zhao D, Yong J, Duo S, Song X, Guo Y, Zhao Y, Qin H, et al: Efficient generation of hepatocyte-like cells from human induced pluripotent stem cells. Cell Res 19: 1233-1242, 2009.

7. Takayama K, Inamura M, Kawabata K, Katayama K, Higuchi M, Tashiro K, Nonaka A, Sakurai F, Hayakawa T, Kusuda Furue M and Mizuguchi H: Efficient generation of functional hepatocytes from human embryonic stem cells and induced pluripotent stem cells by HNF4 $\alpha$ transduction. Mol Ther 20: 127-137, 2012.

8. Zaret KS, Watts J, Xu J, Wandzioch E, Smale ST and Sekiya T: Pioneer factors, genetic competence and inductive signaling: Programming liver and pancreas progenitors from the endoderm. Cold Spring Harb Symp Quant Biol 73: 119-126, 2008.

9. Inamura M, Kawabata K, Takayama K, Tashiro K, Sakurai F, Katayama K, Toyoda M, Akutsu H, Miyagawa Y, Okita $\mathrm{H}$, et al: Efficient generation of hepatoblasts from human ES cells and IPS cells by transient overexpression of homeobox gene HEX. Mol Ther 19: 400-407, 2011

10. Tomizawa M, Shinozaki F, Sugiyama T, Yamamoto S, Sueishi M and Yoshida T: Single-step protocol for the differentiation of human-induced pluripotent stem cells into hepatic progenitor-like cells. Biomed Rep 1: 18-22, 2013.

11. Pol S, Bousquet-Lemercier B, Pave-Preux M, Pawlak A, Nalpas B, Berthelot P, Hanoune J and Barouki R: Nucleotide sequence and tissue distribution of the human mitochondrial aspartate aminotransferase mRNA. Biochem Biophys Res Commun 157: $1309-1315,1988$.

12. Ellinger JJ, Lewis IA and Markley JL: Role of aminotransferases in glutamate metabolism of human erythrocytes. J Biomol NMR 49: 221-229, 2011.
13. Rines AK, Sharabi K, Tavares CD and Puigserver P: Targeting hepatic glucose metabolism in the treatment of type 2 diabetes. Nat Rev Drug Discov 15: 786-804, 2016.

14. Tomizawa M, Shinozaki F, Sugiyama T, Yamamoto S, Sueishi M and Yoshida T: Survival of primary human hepatocytes and death of induced pluripotent stem cells in media lacking glucose and arginine. PLoS One 8: e71897, 2013.

15. Tomizawa M, Shinozaki F, Motoyoshi Y, Sugiyama T, Yamamoto S and Ishige N: An optimal medium supplementation regimen for initiation of hepatocyte differentiation in human induced pluripotent stem cells. J Cell Biochem 116: 1479-1489, 2015.

16. Zhang D,LiJ, Wang F,Hu J, Wang S and Sun Y: 2-Deoxy-D-glucose targeting of glucose metabolism in cancer cells as a potential therapy. Cancer Lett 355: 176-183, 2014.

17. Brito AF, Mendes M, Abrantes AM, Tralhão JG and Botelho MF: Positron emission tomography diagnostic imaging in multidrug-resistant hepatocellular carcinoma: Focus on 2-deoxy-2-(18F)fluoro-D-glucose. Mol Diagn Ther 18: 495-504, 2014.

18. Shan J, Schwartz RE, Ross NT, Logan DJ, Thomas D, Duncan SA, North TE, Goessling W, Carpenter AE and Bhatia SN: Identification of small molecules for human hepatocyte expansion and iPS differentiation. Nat Chem Biol 9: 514-520, 2013.

19. Nakamura T, Teramoto H, Tomita $Y$ and Ichihara A: L-proline is an essential amino acid for hepatocyte growth in culture. Biochem Biophys Res Commun 122: 884-891, 1984.

20. Mitaka T, Sattler CA, Sattler GL, Sargent LM and Pitot HC: Multiple cell cycles occur in rat hepatocytes cultured in the presence of nicotinamide and epidermal growth factor. Hepatology 13: 21-30, 1991.

21. Davies B and Fried M: The L19 ribosomal protein gene (RPL19): Gene organization, chromosomal mapping and novel promoter region. Genomics 25: 372-380, 1995.

22. Tam S, Clavijo A, Engelhard EK and Thurmond MC Fluorescence-based multiplex real-time RT-PCR arrays for the detection and serotype determination of foot-and-mouth disease virus. J Virol Methods 161: 183-191, 2009.

23. Panopoulos AD, Yanes O, Ruiz S, Kida YS, Diep D, Tautenhahn R, Herrerías A, Batchelder EM, Plongthongkum N, Lutz M, et al: The metabolome of induced pluripotent stem cells reveals metabolic changes occurring in somatic cell reprogramming. Cell Res 22: 168-177, 2012.

24. Madonna R, Görbe A, Ferdinandy P and De Caterina R: Glucose metabolism, hyperosmotic stress, and reprogramming of somatic cells. Mol Biotechnol 55: 169-178, 2013.

25. Tomizawa M, Shinozaki F, Motoyoshi Y, Sugiyama T, Yamamoto $\mathrm{S}$ and Ishige N: Hepatocyte selection medium eliminating induced pluripotent stem cells among primary human hepatocytes. World J Methodol 5: 108-114, 2015.

26. Phillips JW, Jones ME and Berry MN: Implications of the simultaneous occurrence of hepatic glycolysis from glucose and gluconeogenesis from glycerol. Eur J Biochem 269: 792-797, 2002. 